4

\title{
Sleep and Next-Day Negative Affect and Suicidal Ideation in Borderline Personality
}

\section{Disorder}

Aleksandra Kaurin ${ }^{\mathrm{a}}$, Garrett Hisler ${ }^{\mathrm{b}}$, Alexandre Y. Dombrovski ${ }^{\mathrm{b}}$, Michael N. Hallquist ${ }^{\mathrm{c}}$ \& Aidan G.C. Wright ${ }^{\mathrm{a}}$

${ }^{a}$ Department of Psychology, University of Pittsburgh

${ }^{\mathrm{b}}$ Department of Psychiatry, University of Pittsburgh School of Medicine

${ }^{\mathrm{c}}$ Department of Psychology and Neuroscience, University of North Carolina, Chapel Hill

(C) 2021, American Psychological Association. This paper is not the copy of record and may not exactly replicate the final, authoritative version of the article. Please do not copy or cite without authors' permission. The final article will be available, upon publication, via its DOI: $10.1037 /$ per0000496

This study was supported by the National Institute of Mental Health (R01 MH048463).

The authors certify that they have no affiliations with or involvement in any organization or entity with any financial interest, or non-financial interest in the subject matter or materials discussed in this manuscript. We are grateful to the patients who took part in this study and to the clinical and research teams who made this work possible.

Correspondence concerning this article should be addressed to Aleksandra Kaurin, Department of Psychology, University of Pittsburgh, 3213 Sennott Square, 210 S. Bouquet St., Pittsburgh, PA, 15260. E-Mail: kaurinaleksandra@pitt.edu 
Abstract

Sleep disturbance is associated with elevated suicidal ideation and negative affect. To date, however, no study has investigated the temporal relationship between sleep and suicidality

29 among those diagnosed with borderline personality disorder (BPD). This preregistered

30 (https://osf.io/4vugk) study tested whether nightly sleep (self-reported sleep duration, sleep onset

31 latency, and subjective sleep quality) represents a (within-person) short-term risk factor for

32 affective dysregulation and increases in suicide risk from day-to-day, as well as whether

33 between-person differences in sleep, negative affect, and suicidality were associated. We used a

34 21-day ecological momentary assessment protocol in a sample of 153 people diagnosed with

35 BPD, 105 of which had a history of serious suicide attempts, and 52 healthy controls $(N=4086$

36 days). We found a within-person association between worse subjective sleep quality and greater

37 next day negative affect. At the between-person level, we found positive relationships between

38 sleep latency and suicidal ideation, and a negative association between subjective sleep quality

39 and negative affect. BPD severity did not significantly moderate the strength of any within-

40 person associations, although BPD was positively associated with average levels of suicidal

41 ideation, sleep latency, and negative affect, and negatively related to subjective sleep quality.

42 These findings suggest that the association of sleep with suicidal ideation and BPD exists largely

43 at the between-persons rather than the within-person level. Disturbed sleep, therefore, seems to

44 largely coincide, rather than specifically contribute to the exacerbation of suicidal crises in BPD. Key words: suicidal ideation; ecological momentary assessment; borderline personality

46 disorder; multilevel structural equation modelling; sleep 
Sleep and Next-Day Negative Affect and Suicidal Ideation in Borderline Personality Disorder

Research suggests that the association between sleep disturbances and suicidal ideation

49 may be potentiated by Borderline Personality Disorder (BPD; Winsper \& Tang, 2014; Winsper

50 et al., 2017). Previous work, however, has returned contradictory results (e.g., Oltmanns \&

51 Oltmanns, 2015), and the temporal, within-person link of sleep and suicidal ideation in BPD has

52 received limited clinical and research attention (Winsper \& Tang, 2014; see Liu et al. (2020) for

53 a recent systematic review and meta-analysis of longitudinal studies). In this study, we address

54 this gap in the literature by asking: Are sleep disturbances contributing factors to exacerbating

55 short-term suicide risk, or an epiphenomenal aspect of BPD? Our analyses are based on a

56 preregistered study protocol (https://osf.io/4vugk), and a 21-day ambulatory assessment

57 procedure, in a carefully sampled population of suicide attempters and non-attempters diagnosed 58 with BPD.

Meta-analytic (Winsper et al., 2017), and narrative reviews (Oltmanns \& Oltmanns, 2015) present strong evidence that BPD is linked to sleep disturbance (Oltmanns, 2019; Winsper

61 et al., 2017). Compared to healthy controls, people diagnosed with BPD experience shorter sleep 62 duration, worse sleep efficiency, and reduced REM latency (e.g., Akiskal et al., 1985;

63 McNamara et al., 1984; Reynolds et al., 1985; Simor \& Horváth, 2013). Yet, inconsistent results 64 within this set of studies make it difficult to articulate clear conclusions regarding the 65 relationship of BPD and individual sleep parameters (Hafizi, 2013; Oltmanns \& Oltmanns, 2015;

66 Winsper et al., 2017). These discrepancies could potentially be attributed to the fact that previous

67 related research has been methodologically diverse and conducted in small samples of

68 phenotypically heterogenous groups of individuals with BPD (Harty et al., 2010; Winsper et al., 69 2017). 
One reason why it is important to understand sleep in BPD is that suicidal ideation,

71 suicidal attempts, and even death by suicide have been linked to a variety of indicators of poor

72 sleep, including insomnia, nightmares, poor subjective sleep quality, short self-reported sleep

73 duration or low sleep efficiency (Bromundt et al., 2013; Grove et al., 2020; Hafizi, 2013;

74 Oltmanns, Weinstein, \& Oltmanns, 2014; Sansone, Edwards, \& Forbis, 2010; Selby, 2013; Wall

75 et al, 2020).

Conventionally, poor sleep has been viewed to be reflective of psychiatric burden, rather

77 than representing a stable risk factor of psychopathology. This view, however, is amended by

78 findings suggesting that sleep disturbance may uniquely contribute to the exacerbation of

79 psychopathological symptoms (Tkachenko et al., 2014; Nutt et al., 2008), which is based on the

80 notion that the successful treatment of primary psychopathology does not automatically seem to

81 improve sleep problems (Thase et al. 2002; Mouchabac et al. 2003; Carney et al. 2007). Some

82 cross-sectional evidence also suggests that elevated insomnia symptoms may account for the link

83 between BPD traits and an increased risk for suicide (DeShong \& Tucker, 2019).

Relatedly, a recent set of findings implies that sleep disruption potentially charts the

85 progression of BPD symptom severity and suicidality by impairing emotional functioning

86 (Balestrieri et al., 2006; Fleischer et al., 2012; Grove et al., 2017; Lereya et al., 2017; Morales-

87 Muñoz et al., 2020; Sjostrom et al., 2009; Wojnar et al., 2009; Wall et al., 2020). On a

88 conceptual level, the Emotional Cascade Model (Selby \& Joiner, 2009) provides one plausible

89 explanatory framework for this relationship. The model suggests that upsetting emotional

90 experiences may be potentiated via poor affect regulatory skills that, in turn, may exacerbate in

91 the context of poor sleep in those with a BPD diagnosis. This positive feedback loop contributes

92 to the escalation of negative affect, which then results in exceptionally aversive emotional states 
93 that are met by impulsive and self-harming behaviors in attempts to soothe those affective

94 experiences. Therefore, understanding the clinical significace of sleep for affective dysregulation

95 is particularly relevant to BPD, because cumulative evidence implies that the within-person

96 association between negative affective states and the severity of suicidal ideation is particularly

97 pronounced in BPD (Kaurin et al., 2020; Links et al., 2007; Mou et al., 2018).

98 What cannot be ascertained from previous, largely cross-sectional studies, however, is

99 whether sleep problems precede, thus exacerbate affective dysregulation and suicidal ideation in

100 BPD, or whether sleep problems largely coincide with BPD. Moreover, correlates obtained from

101 cross-sectional designs may further be driven by individual differences or comorbidity rather

102 than dynamical within-person processes, and cannot be ruled out as concomitants to or

103 epiphenomena of suicidal ideation (Liu et al., 2020). To disambiguate both possibilities,

104 longitudinal studies are needed that are designed to characterize temporal aspects of the

105 relationship between sleep and suicidality (Bernert et al., 2015; Littlewood et al., 2017), both of

106 which fluctuate over time and may influence each other (Ben-Zeev et al., 2012; Kleiman et al.,

107 2017; Lemola et al. 2013; Vallieres et al. 2005).

108 A few longitudinal studies have sought to address this issue, but none of them in BPD

109 samples in particular. For instance, Ribeiro et al. (2012) found that insomnia predicted greater

110 suicidal ideation one month later in a sample of young military recruits, but not vice versa. These

111 findings were replicated by Zuromski et al. (2017) in an online sample of individuals endorsing

112 lifetime experience of suicidal behavior. Additionally, a one week-long ecological momentary

113 assessment study reported that subjective and actigraphic sleep duration as well as subjective

114 sleep quality predicted next day ideation (Littlewood et al., 2019). Again, suicidal ideation did

115 not predict next night sleep. Finally, a unidirectional relationship was reported in which 
116 nightmares significantly predicted post-sleep self-harm via increased daytime negative affect in a 117 sample of university students (Hochard et al. 2015).

118 Although it appears from this brief review that disturbed sleep is prospectively linked to

119 elevated levels of suicide risk, it should be noted that the majority of studies assessed sleep

120 disturbance as a long-term risk factor (Liu et al., 2020). Thus, these studies generally provide

121 limited insight into the temporal association between sleep, suicidal ideation, and negative affect,

122 and do not permit disentangling individual difference level associations from the estimates of

123 temporal associations (e.g., person-centering day-level predictors; Bolger \& Laurenceau, 2013).

124 In fact, a recent meta-analysis concluded that sleep disturbance prospectively predicts suicide

125 outcomes, but more work is needed to examine sleep as a short term risk factor and to

126 differentiate the associations of acute from chronic sleep disturbance (Liu et al., 2020).

127 Therefore, it remains unclear to what degree disrupted sleep predicts short-term suicide risk.

128 Narrowing this gap is of substantial clinical importance, because clinical risk assessments are

129 focused on determining if individuals may engage in suicidal behavior in the near future (Liu et

130 al., 2020).

131 To this end, the present study expands upon prior research by disentangling, and then

132 separately estimating the day-level and person-level associations among sleep, negative affect,

133 and suicidal ideation. Such methodological distinction is key to the question whether poor sleep

134 largely coincides with BPD, or significantly contributes to the short-term exacerbation of BPD

135 symptoms (including negative affectivity and suicidality) from day to day. As such, we aim to

136 contribute to a better understanding of whether temporal changes in sleep predict short-term

137 changes of suicide risk, and whether that relationship is accounted for by negative affect. 
Although previous research has found sleep disturbance to contribute to elevated negative

140 affect, and suicidal ideation, no study has investigated the temporal relationship between sleep

141 and suicidality in BPD, whether negative affect accounts for this relationship, nor directly

142 examined the separate day-level and person-level associations. The current preregistered

143 (https://osf.io/4vugk) study responds to a recent call for more methodologically rigorous studies

144 of the sleep-suicidal ideation relationship (Bernert et al., 2015; Littlewood et al., 2017; Liu et al.,

1452020 ) by investigating the temporal relationships among sleep, negative affect, and suicidal

146 ideation using a 21-day ecological momentary assessment protocol in a sample of 142 people

147 diagnosed with BPD, 98 of which had a history of serious suicide attempts, and 49 healthy

148 controls. This sampling approach increases the likelihood of participants reporting suicidal

149 ideation during the study period, because past suicide attempts represent the most reliable

150 predictors of future suicide attempts (O’Connor \& Nock, 2014). Moreover, the design of this

151 study allows to improve limitations in the suicide literature by utilizing a brief follow-up period

152 and focusing on sleep as a less studied short-term risk factor for suicidal ideation in BPD.

153 Based on previous work reviewed above, we set out to disambiguate whether sleep

154 problems antecede and potentially aggravate short-term suicidal ideation in BPD, or whether

155 disturbed sleep and suicidal ideation largely coincide with BPD. We tested a series of hypotheses

156 related to whether nightly sleep predicted next-day suicidal ideation or negative affect, and

157 whether BPD severity moderated the dynamic links among those variables as they fluctuate,

158 within-person, from day to day. Specifically, our hypotheses were:

159 Tests of Within-Person Associations

160 H1a: Worse nightly sleep would predict greater next-day suicidal ideation.

161 H1b: Worse nightly sleep would predict greater next-day negative affect. 
H1c: The within-person association between worse sleep and next-day suicidal ideation would be accounted for by the next-day negative affect.

\section{Cross-Level Interaction Effects}

165 H2a: BPD features would magnify the within-person association between sleep 166 disturbance and suicidal ideation.

167 H2b: BPD features would magnify the within-person association between sleep 168 disturbance and negative affect.

169 Our hypotheses are presented in simplified form for clarity. Estimated models included 170 covariates (i.e., between-level: age, gender; within-level: day in study, and weekday vs.

171 weekend). Sleep onset latency, shorter sleep duration, and worse subjective quality were

172 examined each night and models were conducted separately for each sleep parameter to avoid 173 multicollinearity. Of note, we have tested the association between negative affect and suicidality

174 at both, the within- and between-person level in previous analyses. Results from these analyses 175 revealed that negative affect significantly predicts daily levels of suicidality as operationalized in 176 the models below (Kaurin et al., 2020).

177 Recent meta-analytic evidence suggests that the link between disturbed sleep and BPD 178 features is likely independent of comorbid depression (Winsper et al., 2017). However, given the 179 importance of persistent negative affectivity for the prediction of suicidal behavior (Zanarini et 180 al., 2016), we exploratorily probed the robustness of our analyses by controlling for current 181 depressive symptoms at the between-person level (osf.io/cxy9b). 
All study procedures were approved by the Institutional Review Boards of the University 184 of Pittsburgh (STUDY19050210), and all data, and analyses have been made publicly available 185 at the OSF.

186 Sample. Participants were drawn from a longitudinal study, and were recruited from in187 and outpatient clinics as well as from the nearby community by advertisement. At enrollment, 188 participants had to be between 18 and 45 years and discharged from any inpatient treatment 189 setting. Exclusion criteria included a lifetime diagnosis of any psychotic or bipolar disorder, 190 clinical evidence of organic brain disease, physical disorders or treatments with known 191 psychiatric consequence (e.g., lupus, steroids), and IQ<70 measured by WAIS. A more detailed 192 description of the parent study protocol has been reported elsewhere (Soloff et al., 2017). The 193 sample comprised 153 individuals diagnosed with BPD and 52 healthy control participants 194 (mean age: $33.64, \mathrm{SD}=9.50$; range $20-60$ years; $80 \%$ female; $74 \%$ Caucasian, $12 \%$ African 195 American, 5\% Asian, 5\% Multi-Race, 4\%Pacific Islander or non-specified). Of the 153 BPD 196 patients, $n=35$ had a history of high-lethality, $n=70$ had past low-lethality past suicide attempts, 197 and $n=48$ reported no past suicide attempts. As preregistered, to increase the consistency of our 198 EMA measurements, participants with less than 5 days of sleep were excluded from data 199 analyses. Moreover, data on bed and waketime variables were excluded from analyses if 200 identified as an outlier at 4 standard deviations from the mean. This resulted in a final sample 201 size of $N=201$ participants, which did not significantly differ from the original sample in key 202 outcomes.

203 The diagnosis of BPD was based on the International Personality Disorder Examination 204 (IPDE; Loranger et al., 1996), and participants had to meet probable or definite criteria for a 205 lifetime diagnosis. Participants diagnosed with BPD were further divided into two groups based 
on history of reported suicide attempts. Suicide attempters were required to have clinically

207 significant attempts, defined by a score of $>1$ on the Beck Lethality Scale (Beck, Beck, \&

208 Kovacs, 1975). For participants with multiple attempts, data for the highest-lethality attempt

209 were used.

210 Participants of the healthy control group and were required to have no lifetime Axis I or

211 II diagnoses determined by the Structured Clinical Interview for DSM-IV or IPDE. As

212 preregistered, participants from the three groups were merged to represent a range of symptom

213 severity, which was further specified with the Personality Assessment Inventory-Borderline

214 Scale (PAI-BOR; Morey, 1991), which demonstrated excellent (alpha=.81) reliability in our

215 sample. The PAI-BOR contains four subscales that tap core features of BPD: affective

216 instability, identity problems, negative relationships, and self-harm. The self-harm subscale taps

217 impulsivity and impulsive behaviors more broadly and is not limited to self-harm behaviors.

218 Momentary Assessments. Participants completed a 21-day EMA protocol within pre-

219 defined time windows, using the MetricWire smartphone application, which randomly reminded

220 them to complete surveys via push notifications approximately six times per day. This resulted in

$221 N=4086$ days (based on $N=24916$ momentary assessments), with an average number of 19.9 days

222 per participant, ranging from 1-22 days overall. Based on data preregistered exclusion

223 procedures detailed above, the final sample comprised $N=4076$ days and an average number of

22420 days per participant.

225 Each morning, participants reported on last night's sleep characteristics. Specifically,

226 they reported what time they went to bed the prior night, how long it took them to fall asleep

227 (i.e., sleep onset latency), what time they woke up, and the quality of the prior night sleep (i.e., 228 subjective sleep quality). 
Sleep duration was calculated from bedtime, waketime, and sleep onset latency reports.

230 First, bedtime and waketime reports were checked for reporting errors (e.g., participants

231 reporting a bedtime of 10:00 instead of 22:00) and either transformed to their appropriate time or 232 were removed from the data. After addressing potential bed and waketime reporting errors, sleep

233 duration was calculated by the difference, in hours, between self-reported waketime and bedtime.

234 In order to calculate sleep duration, we added 24 hours to the waketime and subtracted

235 bedtime from the transformed waketime variable. To illustrate, if a participant reported a

236 bedtime of 23:00 and a waketime of 8:00, the waketime of 8:00 would be transformed to 32:00

237 by adding 24 hours. Then, the bedtime of 23:00 was subtracted from 32:00 to calculate a sleep 238 duration of 9 hours.

239 Sleep onset latency was reported on a scale in which each response was a 15 chunk of 240 time (e.g., 1 = "under 15 minutes", 2 = "15-30 minutes"). This sleep onset latency scale ranged

241 from "under 15 minutes" to "1 hour 45 minutes to 2 hours." Sleep onset latency was recoded so 242 that participant responses reflected the mean of the selected response option (e.g., responses of

243 between 15-30 minutes were recoded to equal 22.5 minutes). Note that the 15 -minute interval of 2441 hour 30 minutes to 1 hour 45 minutes was erroneously left out of the scale during data 245 collection. Subjective sleep quality was measured with the question "Last night my sleep quality 246 was...", based on a five-point rating scale, ranging from 1 ("very poor") 5 ("very good").

247 Participants reported how strongly they experienced negative (i.e., nervous, sad, irritated, angry; $248 \omega_{\text {within-person }}=.81 ;$ bretween-person $\left.=.91\right)$ derived from the Positive and Negative Affect Schedule 249 (Watson et al., 1988), on a scale from 0 ("Not at All") to 100 ("Extremely") for each adjective. 250 Suicidal ideation was assessed with six dichotomous items (1=yes, $0=$ no; range: $0-5.4)$ 251 derived from the suicidal ideation subscale of the Columbia-Suicide Severity Rating Scale (C- 
252 SSRS; Posner et al., 2008). All items were used to form a daily mean score of suicidal intent 253 severity, and read as follows: "Have you wished you were dead or wished you could go to sleep 254 and not wake up?", "Have you actually had any thoughts of killing yourself?”, "Have you been 255 thinking about how you might do this?", "Have you had these thoughts and had some intention 256 of acting on them?", "Have you started to work out or worked out the details of how to kill 257 yourself?", "Do you intend to carry out this plan?" $(\omega$ within= .67 ; $\omega$ between= .83$)$. The 258 examination of intraclass correlation returned that approximately $60 \%$ of the variability in ratings 259 of suicidal ideation was explained by between-person differences $(\mathrm{ICC}=.601)$.

On $11.4 \%$ of days participants reported suicidal ideation at least once during the day.

261 More specifically, participants in the BPD group endorsed suicidal ideation on $6.2 \%$, and suicide 262 attempters, on $19.5 \%$ of all study days. Among the healthy controls, one participant endorsed a 263 single episode of suicidal ideation.

264 Data Analysis. As preregistered, multi-level structural equation models (MSEMs) were

265 used to test our hypotheses of the links between daily sleep, affect and suicidal ideation. All 266 models were estimated in Mplus (version 8.4; Muthén \& Muthén, 1998-2019). We used

267 Bayesian estimation because it provides a true latent decomposition into within- and between-

268 person variance for both the predictors and outcome variables when random effects are specified.

269 Significance for all model parameters was based on 95\% Credibility Intervals (CIs), with CIs

270 that excluded zero indicative of a parameter that differed significantly from zero. Figure 1

271 provides a hypothesis-by-hypothesis schematic overview of the estimated models, along with 272 path annotations as referenced in the results section.

273 Total variance in the observed variables of sleep and suicidal ideation was decomposed 274 into their constituent pieces. Because sleep is a daily level variable nested within people, it was 
275 decomposed into its day-level and person-level variables using latent decomposition.

276 Accordingly, both assessments of suicidal ideation and negative affect were averaged over each

277 day, and decomposed into their day-level, and person-level latent variables. This decomposition

278 is akin to the partitioning of variance that occurs with the outcome variable in standard

279 multilevel modeling, however in MSEM it is conducted for both outcomes and predictors that

280 have repeated observations within a person. The between person-level latent variables are an

281 estimate of an individual's average (i.e., random intercepts), and resemble the portion of variance

282 that is usually captured in cross-sectional studies. The day-level variables reflect the deviations

283 from their average on a given day, for that person, respectively.

284 Based on our preregistered hypotheses, two sets of models were estimated with nightly

285 sleep (i.e., latency, duration, quality) predicting daily suicidal ideation or negative affect. Model

286 Set $1(H 1 a)$ regressed daily suicidal ideation on nightly sleep at Level 1 (i.e., sleep and daily

287 suicidal ideation), and Level 2 (i.e., associations among individual differences in average sleep

288 parameters and suicidal ideation). Model Set $2(H 1 b)$, regressed daily negative affect on nightly

289 sleep at both levels. To test H1c, Model Set 3 combined Models 1 and 2 by introducing negative

290 affect as intermediate variable for the relationship between sleep and suicidal ideation at Level 1

291 and Level 2.

292 To test the second set of hypotheses, Models $4(H 2 a)$ and $5(H 2 b)$ introduced BPD

293 symptom severity as moderator of the link between sleep and suicidal ideation, or sleep and

294 negative affect (i.e., cross-level interactions), while also predicting individual differences in

295 ratings of one's nightly sleep, negative affect and suicidal ideation.

296 Age and gender $(0=$ female; $1=$ male $)$ were modeled as covariates at the between-person

297 level, and random slopes and intercepts were regressed on these covariates. Study day and 
298 weekend (vs. weekday) were modeled as covariates at the within-person level to control for any

299 confounding influence of time and weekend effects. No prior information about parameter values

300 were specified (i.e., we used non-informative/diffuse priors that are the default in MPlus).

301 Missing data was assumed to be missing at random. A Bayesian approach to SEM uses all

302 available data in estimation: with increasingly large samples, it provides similar results to Full

303 Information Maximum Likelihood to address missing data (Asparouhov \& Muthén, 2010).

304 Along with other parameters not reported in the tables (e.g., residual variances),

305 covariances among between-persons variables are not depicted in the diagrams, but full

306 specifications and detailed output from all models can be found online at osf.io/cxy9b. Table 1

307 summarizes pooled within-person correlations among the variables along with correlations

308 among the random intercepts (i.e., person averages) at the between-person level.

\section{Results}

\section{Model Set 1: Does nightly sleep predict next-day suicidal ideation?}

311 Results for models examining the effects of sleep (i.e., sleep latency, duration, and

312 quality) on next-day suicidal ideation can be found in the first column of Table 2. None of the

313 three model specifications returned a significant link between sleep and next day suicidal

314 ideation at the within-person level. That is, on days when participants reported worse than

315 average nightly sleep, they did not also report greater suicidal ideation. At the between-person

316 level, however, we found a relationship between sleep latency and suicidal ideation suggesting

317 that participants who reported taking a longer time to fall asleep also reported higher average

318 levels of suicidal ideation.

319 Model Set 2: Does nightly sleep predict next-day negative affect? 
Key estimates for models testing the effects of sleep (i.e., sleep latency, duration, and

321 quality) on next-day negative affect are detailed in the central column of Table 2. Overall, we

322 only found a significant positive within-person link between subjective sleep quality and next

323 day negative affect. Thus, on average, when individuals reported worse nightly subjective sleep

324 quality, they also tended to report elevated levels of negative affect on the subsequent day.

325 At the between-persons level, a positive relationship emerged for negative affect and

326 sleep latency, and a negative association for subjective sleep quality and negative affect. That is,

327 those who generally tended to report longer sleep onset latency and worse subjective sleep

328 quality, also reported higher levels of negative affect on average.

\section{Model 3: Does negative affect explain the relation between nightly sleep and next-day}

\section{0 suicidal ideation?}

331 As noted in our preregistration, our aim was to combine both Model 1 and Model 2 to test

332 whether negative affect would account for the relationship between poor nightly sleep and

333 suicidal ideation. Unsurprisingly given the nonsignificant direct associations between sleep

334 variables and suicidal ideation at the within-person level, negative affect did not statistically

335 mediate these associations (see right column of Table 2, Model 3).

336 Model 4: Does BPD severity moderate the associations among sleep, negative affect, and

\section{7 suicidal ideation?}

338 Finally, we tested whether BPD severity features moderated the paths of the within-

339 person Models 1 and 2. All within-person paths exhibited significant variability across

340 individuals. Individual differences in the strength of these within-person paths were regressed on

341 BPD severity, sex, and age. Coefficients from these models are displayed in Table 3, organized 
342 by sleep variables. Results indicated that BPD severity had no significant effect on any within-

343 person pathway, although it did have associations with between-person differences.

Specifically, BPD severity was positively associated with individual differences in

345 suicidal ideation, except for the model where suicidal ideation was regressed on sleep latency.

346 BPD severity was also positively related to sleep latency and negatively to subjective sleep

347 quality. Additionally, in models were negative affect was regressed on sleep, we found that BPD

348 severity was associated with greater negative affect (see Table 3 for an overview).

\section{Discussion}

350 Whereas previous studies reported long-term associations between disturbed sleep and

351 suicide risk, it remained unclear whether these associations were driven by individual differences

352 or dynamic within-person processes. Therefore, sleep disturbances could not be ruled out as

353 concomitant to or an epiphenomenon of BPD, and to what degree disrupted sleep predicts short-

354 term suicide risk in contrast (see Liu et al., 2020 for a systematic review and meta-analysis of

355 longitudinal studies). To narrow this gap in the literature, we obtained data from a large, well-

356 characterized BPD sample oversampled for past suicide attempts, and preregistered our analyses.

357 In contrast to our first set of preregistered hypotheses, we did not find a significant

358 within-person association between daytime suicidal ideation and self-reports of how a participant

359 slept the night before. Overall, this pattern contrasts with sparse previous evidence that prior

360 sleep disruption predicts suicidal ideation (Hochard et al., 2015; Ribeiro et al., 2012; Zuromski et

361 al., 2017). It particularly contrasts with Littlewood and colleagues (2019) who found that both

362 self-reported sleep duration and subjective sleep quality predicted greater next-day suicidal

363 ideation. One explanation may be that these prior studies did not appear to explicitly disentangle

364 and separately model within-person and between-person associations between sleep and suicidal 
365 ideation. Moreover, the use of community or non-BPD samples in these studies limits the ability

366 to generalize results to BPD samples, like the sample reported in this study. It is also worth

367 noting that the current study collected substantially more repeated assessments per person than

368 most prior studies, and obtained a significantly larger sample size (Hochard et al., 2015;

369 Littlewood et al., 2019; Ribeiro et al., 2012), affording more accuracy when approximating links

370 between BPD, suicidal ideation and sleep (e.g., Oltmanns, 2019).

371 At the between-person level, however, we found that people who generally take longer to

372 fall asleep also tended to report greater suicidal ideation. The relationship between sleep latency

373 and suicidal ideation could be reflective of more general, aberrant self-regulatory processes in

374 BPD that are shared, potentially etiological and maintaining factors of poor subjective sleep

375 quality in BPD. However, because these links emerged at the between- rather than the within-

376 person level, sleep disturbances likely reflect concomitants or epiphenomena of BPD rather than

377 a stable risk factor (Liu et al., 2020). In that regard, it is reasonable to assume that effects of

378 sleep on BPD symptom exacerbation unfold over longer periods of time, and thus more likely

379 resemble stable predispotions to affective dysregulation.

380 Accordingly, tests of the second set of hypotheses revealed that at the between-person

381 level, people with greater average negative affect had longer sleep onset latency and worse

382 subjective sleep quality on average. Moreover, a significant within-person link between

383 subjective sleep quality and negative affect emerged, wherein a night of better quality of sleep

384 than typical predicted less than usual next day negative affect. Importantly, this effect may be

385 interpreted as a check of our study's ability to detect within-person relationships in general.

386 Overall, these findings generally replicate findings that sleep and negative affect are linked at

387 both the within- and between-person level (Kahn et al., 2013; Palmer \& Alfano, 2017). They also 
converge with evidence that a number of symptoms and behaviors central to BPD are known to disrupt sleep patterns (Fleischer et al., 2012; Winsper \& Tang, 2014). Relatedly, Harvey et al. (2011) proposed that it is important to address both sleep disturbance and emotion dysregulation,

391 in treatment to produce durable treatment gains. For instance, helping to stabilize sleep prior to

392 addressing emotion regulation skills might facilitate skill acquisition and improve overall

393 treatment outcome (see also Nutt et al., 2008).

Regarding our third hypothesis, we did not find that negative affect accounted for the

within-person link between sleep and suicidal ideation. Wall et al (2020) reported a similar pattern in cross-sectional data, where adolescent BPD and affective dysregulation were significantly related to sleep disturbance, but affective dysregulation did not account for the link between sleep disturbance and BPD.

Finally, tests of our second set of hypotheses revealed, that none of the significant within400 person links was moderated by BPD severity. In line with previous work (Winsper et al., 2017), 401 however, we found that BPD severity was generally positively associated with suicidal ideation, 402 and sleep latency, while its was negatively associated with subjective sleep quality. Additionally, 403 in models where negative affect was regressed on sleep, we found that BPD severity was 404 positively related to negative affect. Thus, people with greater severity of BPD had worse sleep, 405 greater negative affect, and greater suicidal ideation on average. However, these participants did not have stronger within-person links among these variables. Moreover, these effects were

407 unique to BPD, even after controlling for depression, which we demonstrated in an exploratory, 408 not preregistered set of analyses (ㅇf.io/cxy9b). Overall, these findings suggest that the associations of sleep with suicidal ideation and 
411 who experience more negative affect in general also tend to report lower subjective sleep quality

412 or greater sleep latency. Additionally, those who reported longer average sleep latency also

413 endorsed higher levels of suicidal ideation. In addition, poor sleep and greater suicidal ideation

414 tended to characterize individuals higher in BPD severity rather than poor sleep exacerbating

415 suicidal ideation and negative emotions in BPD patient populations. It is possible that the pattern

416 of results reported in this study reflects more general self-regulatory impairments in BPD, that

417 have the potential to spill over into disturbed nightly sleep (e.g., Harty et al., 2010; Selby et al.,

418 2013) that may largely coincide, though not specifically contribute to the exacerbation of suicidal

419 crises in BPD.

\section{Study Limitations}

421 The current research has limitations that are important to consider. First, findings are

422 based upon self-report data which may upwardly bias associations due to common method

423 variance (Podsakoff et al., 2003). Additionally, self-reports of sleep demonstrate moderate

424 convergence with actigraphy and polysomnographic measures of sleep and future research

425 should strive to use other methods to measure sleep. Along these lines, participant raw reports of

426 bedtime and waketime, which were used to calculate sleep duration, had reporting errors (a

427 frequent, yet infrequently discussed problem with self-reports of bed and waketimes) that either

428 had to be corrected or resulted in data loss. Using an actigraphic measure of sleep would avoid

429 such reporting errors, (though it is still prone to other data cleaning problems, see Fekedulegn et

430 al., 2020). Despite the limitation of using self-reported sleep data, it should be noted that

431 subjective sleep quality is a unique sleep characteristic that can only be measured by self-report

432 and which is an important, distinct element of sleep that is tied to health and functioning

433 (Buysse, 2014). 
Even in real-world, every day contexts and representative, high-risk populations, the base rate of momentary endorsements of suicidal ideation is low, and the levels reported in this

436 sample were slightly higher than averages reported in previous studies (7.8\% of reports in Husky

437 et al., 2014). This low variance in suicidal ideation likely reduced the ability to detect and

438 downwardly biased associations between sleep and suicidal ideation. Thus, to increase the

439 reliability of our statistical models, we averaged momentarily assessed suicidal ideation over

440 each day. This allowed us to create a detailed measure of suicidal ideation severity,

441 encompassing a wide range of suicidal thoughts and potential behaviors that no previous

442 momentary study has assessed. Future studies could, therefore, explore whether different item

443 and scaling choices would affect response rates, and also, which temporal resolution would yield 444 most reliable estimates.

445 Further, our findings at the between-person level are cross-sectional in nature and 446 therefore preclude causal conclusions. Thus, while an individual's BPD severity and average 447 levels of sleep, negative affect, and suicidal ideation all tended to be associated with each other, 448 it is unclear whether any of these characteristics precede or cause one another. There is limited 449 evidence supporting the notion that sleep problems may be evident before the development of 450 BPD and contribute to its etiology (Lereya et al., 2017; Selby et al., 2013; Wall et al., 2020).

451 There is also tentative evidence to suggest that ongoing sleep problems can contribute to the 452 chronicity of the disorder (Plante et al., 2013). Intensive longitudinal studies focused on more 453 chronic levels of these characteristics, across different developmental stages are needed to 454 determine how poor sleep and short-term fluctuations in suicidal ideation relate to each other in 455 daily life. 
Although we have probed and illustrated the robustness of our study results via sensitivity analyses controlling for concurrent depression (osf.io/cxy9b), the present study is - to some extent - limited by a comprehensive control strategy of important confounders of sleep

459 distrubances, such as other relevant comorbid disorders or socio-economic features (e.g.,

460 working status). Data derived from ambulatory assessment protocols allow to characterize

461 temporal aspects of the relationship between sleep, affect and suicidality as they fluctuate over

462 time as well as their reciprocal influence. Cross-sectional analyses, in contrast, focus on

463 epiphenomena of suicidal ideation. Thus, although it is plausible to assume that it is

464 predominantly the latter relationship that is more susceptible to biases by unobserved confounds,

465 future studies should investigate whether the dynamic coupling between variables of interest may 466 be weakened or enhanced in the context of theoretically justifiable control variables.

468 of nightmares, which are known to occur frequently in BPD (Winsper et al., 2017). This is a

469 critical limitation, because preliminary evidence suggests a prospective association between

470 nightmares and BPD symptoms based on intensive longitudinal data in a small sample (Selby et

471 al., 2013) as well as over longer developmental periods (Lereya et al., 2016). There is also

472 growing evidence showing that nightmares are associated with increased risk of suicidal

473 behavior or self-harm in clinical and community samples (e.g., Hochard et al., 2015; Russel,

474 Rasmussen, \& Hunter, 2017), independently of a history of traumatic experience (Sandmann et

475 al, 2017). Possibly, nightmares aggravate daytime affective dysregulation which may manifest in 476 symptom exacerbation; alternatively, nightmares may also be reflective of daily affect

477 dysregulatory experiences (Selby et al., 2013). Thus, more research is needed to clarify the 478 direction and magnitude of the relationship between nightmares and affect-regulatory processes 
479 in daily life, and how each of these processes as well as their interactive effects may contribute to 480 states of heightened risk for death by suicide.

\section{Conclusion}

This is the first preregistered study examining sleep as a short-term risk factor of suicidal

483 ideation in a well-characterized BPD sample. We believe that it represents an important first step

484 toward rigorous research on how sleep and suicide are related on a narrow timescale in BPD (see

485 also Oltmanns, 2019), and emphasize the importance of disentangling within- and between-

486 person associations in this context. Because our results suggest that the link between sleep

487 disturbance and suicidal ideation in BPD largely exists at the individual difference level rather

488 than the within-person level, it is reasonable to assume that sleep disturbance generally emerges

489 as part of the disorder. Those who experience generally elevated levels of negative affect, also

490 tend to report lower subjective sleep quality or greater sleep latency, while those who experience

491 generally more pronounced levels of sleep latency, also endorse higher levels of suicidal

492 ideation. In other words, poor sleep and greater suicidal ideation tend to characterize individuals

493 higher in BPD severity rather than poor sleep exacerbating suicidal ideation and negative

494 emotions in BPD. Possibly, effects of sleep on BPD symptom exacerbation unfold over longer

495 periods of time, which is why they phenomenologically resemble stable predispositions to

496 affective dysregulation, and more general self-regulatory impairments likely manifest as

497 disturbed nightly sleep in BPD.

498 Disturbed sleep, therefore, seems to largely coincide, rather than specifically contribute to 499 the exacerbation of suicidal crises in BPD. 


\section{References}

Akiskal, H.S., Yerevanian, B.I., Davis, G.C., King, D., \& Lemmi, H. (1985). The nosologic status of borderline personality: Clinical and polysomnographic study. The American Journal of Psychiatry, 142, 192-198.

Asparouhov, T., \& Muthén, B. (2010). Bayesian analysis of latent variable models using Mplus.

Balestrieri, M., Rucci, P., Sbrana, A., Ravani, L., Benvenuti, A., Gonnelli, C., ... \& Cassano, G. B. (2006). Lifetime rhythmicity and mania as correlates of suicidal ideation and attempts in mood disorders. Comprehensive psychiatry, 47(5), 334-341.

Beck, A.T., Beck, R., \& Kovacs, M. (1975). Classification of suicidal behaviors: I. Quantifying intent and medical lethality. The American Journal of Psychiatry, 132(3), 285-287.

Ben-Zeev, D., Young, M.A., \& Depp, C.A. (2012). Real-time predictors of suicidal ideation: mobile assessment of hospitalized depressed patients. Psychiatry Research, 197(1-2), 5559.

Bernert, R.A., Kim, J.S., Iwata, N.G., \& Perlis, M.L. (2015). Sleep disturbances as an evidence-based suicide risk factor. Current Psychiatry Reports, 17(3), 15.

Bolger, N., \& Laurenceau, J.P. (2013). Intensive longitudinal methods: An introduction to diary and experience sampling research. Guilford Press.

Bromundt, V., Wirz-Justice, A., Kyburz, S., Opwis, K., Dammann, G., \& Cajochen, C. (2013). Circadian sleep-wake cycles, well-being, and light therapy in borderline personality disorder. Journal of Personality Disorders, 27(5), 680-696.

Buysse, D.J. (2014). Sleep health: can we define it? Does it matter?. Sleep, 37(1), 9-17.

DeShong, H.L., \& Tucker, R.P. (2019). Borderline personality disorder traits and suicide risk: 
The mediating role of insomnia and nightmares. Journal of Affective Disorders, 244, 8591.

Fekedulegn, D., Andrew, M.E., Shi, M., Violanti, J.M., Knox, S., \& Innes, K.E. (2020). Actigraphy-Based Assessment of Sleep Parameters. Annals of Work Exposures and Health, 64(4), 350-367.

Fleischer, M., Schäfer, M., Coogan, A., Häßler, F., \& Thome, J. (2012). Sleep disturbances and circadian CLOCK genes in borderline personality disorder. Journal of Neural Transmission, 119(10), 1105-1110.

Grove, J.L., Smith, T.W., Carlson, S.E., Bryan, C.J., Crowell, S.E., Czajkowski, L., ... \& Parkhurst, K. (2020). Prospective association between suicide cognitions and emotional responses to a laboratory stressor: The mediating role of nightly subjective sleep quality. Journal of Affective Disorders, 265, 77-84.

Grove, J.L., Smith, T.W., Crowell, S.E., \& Ellis, J.H. (2017). Preliminary evidence for emotion dysregulation as a mechanism underlying poor sleep quality in borderline personality disorder. Journal of Personality Disorders, 31(4), 542-552.

Hafizi, S. (2013). Sleep and borderline personality disorder: A review. Asian Journal of Psychiatry, 6(6), 452-459.

Harty, L., Forkner, R.D., Thompson, A., Stuewig, J., \& Tangney, J.P. (2010). Are inmates’ subjective sleep problems associated with borderline personality, psychopathy, and antisocial personality independent of depression and substance dependence?. Journal of Forensic Psychiatry \& Psychology, 21(1), 23-38.

Hochard, K.D., Heym, N., \& Townsend, E. (2015). The unidirectional relationship of nightmares on self-harmful thoughts and behaviors. Dreaming, 25(1), 44-58. 
Husky, M., Olié, E., Guillaume, S., Genty, C., Swendsen, J., \& Courtet, P. (2014). Feasibility and validity of ecological momentary assessment in the investigation of suicide risk. Psychiatry Research, 220(1-2), 564-570.

Kahn, M., Sheppes, G., \& Sadeh, A. (2013). Sleep and emotions: bidirectional links and underlying mechanisms. International Journal of Psychophysiology, 89(2), 218-228.

Kaurin, A., Dombrovski, A., Hallquist, M., \& Wright, A.G. (2020, July 22). Daily Suicidal Surge and Attempted Suicide in Borderline Personality Disorder. https://doi.org/10.31234/osf.io/hswea

Kleiman, E.M., Turner, B.J., Fedor, S., Beale, E.E., Huffman, J.C., \& Nock, M.K. (2017). Examination of real-time fluctuations in suicidal ideation and its risk factors: Results from two ecological momentary assessment studies. Journal of Abnormal Psychology, 126(6), 726-738.

Lemola, S., Ledermann, T., \& Friedman, E.M. (2013). Variability of sleep duration is related to subjective sleep quality and subjective well-being: an actigraphy study. PloS one, 8(8), e71292.

Lereya, S.T., Winsper, C., Tang, N.K., \& Wolke, D. (2017). Sleep problems in childhood and borderline personality disorder symptoms in early adolescence. Journal of Abnormal Child Psychology, 45(1), 193-206.

Links, P.S., Eynan, R., Heisel, M.J., Barr, A., Korzekwa, M., McMain, S., \& Ball, J.S. (2007). Affective instability and suicidal ideation and behavior in patients with borderline personality disorder. Journal of Personality Disorders, 21(1), 72-86.

Littlewood, D.L., Kyle, S.D., Carter, L.A., Peters, S., Pratt, D., \& Gooding, P. (2019). Short 
sleep duration and poor sleep quality predict next-day suicidal ideation: an ecological momentary assessment study. Psychological Medicine, 49(3), 403-411.

Liu, R. T., Steele, S. J., Hamilton, J. L., Do, Q. B., Furbish, K., Burke, T. A., ... \& Gerlus, N. (2020). Sleep and suicide: A systematic review and meta-analysis of longitudinal studies. Clinical Psychology Review, 101895.

Loranger, A., Sartorius, N., \& Janca, A. (1996). Assessment and diagnosis of personality disorders: The International Personality Disorder Examination (IPDE). New York, NY: Cambridge University Press. Journal of Psychiatry, 141, 182-186.

Morales-Muñoz, I., Broome, M. R., \& Marwaha, S. (2020). Association of Parent-Reported Sleep Problems in Early Childhood With Psychotic and Borderline Personality Disorder Symptoms in Adolescence. JAMA Psychiatry. Published online July 01, 2020.

Morey, L. C. (1991). Personality assessment screener. Odessa, FL: Psychological Assessment

\section{Resources.}

Mou, D., Kleiman, E.M., Fedor, S., Beck, S., Huffman, J.C., \& Nock, M.K. (2018). Negative affect is more strongly associated with suicidal thinking among suicidal patients with borderline personality disorder than those without. Journal of Psychiatric Research, 104,

588 Muthén, L., \& Muthén, B. (2019). Mplus User’s Guide (2019). Muthén \& Muthén: Los Angeles, $C A$.

590 Nutt, D., Wilson, S., \& Paterson, L. (2008). Sleep disorders as core symptoms of 

depression. Dialogues in Clinical Neuroscience, 10(3), 329-336.

O'Connor, R.C., \& Nock, M.K. (2014). The psychology of suicidal behaviour. The Lancet Psychiatry, 1(1), 73-85.

594 Oltmanns, J.R. (2019). Personality Disorders, Maladaptive Personality Traits, and Sleep Problems: Findings and Future Directions. In Sleep, Personality, and Social Behavior (pp. 239-254). Springer, Cham.

Oltmanns, J.R., \& Oltmanns, T.F. (2015). Borderline personality pathology, polysomnography, and self-reported sleep problems: a review. Current Sleep Medicine Reports, 1(2), 141-

Oltmanns, J.R., Weinstein, Y., \& Oltmanns, T.F. (2014). Borderline personality pathology and insomnia symptoms in community-dwelling older adults. Personality and Mental review. Sleep Medicine Reviews, 31, 6-16. Health, 8(3), 178-187.

Palmer, C.A., \& Alfano, C.A. (2017). Sleep and emotion regulation: an organizing, integrative

Pandi-Perumal, S.R., Monti, J.M., Burman, D., Ramanujam, K., BaHammam, A.S., Spence, D.W., Brown, G.M \& Narashimhan, M. (2020). Clarifying the role of sleep in depression: A narrative review. Psychiatry Research, 113239.

611 Podsakoff, P.M., MacKenzie, S.B., Lee, J.Y., \& Podsakoff, N.P. (2003). Common method biases in behavioral research: a critical review of the literature and recommended remedies. Journal of Applied Psychology, 88(5), 879-903. 
614 Posner, K., Brent, D., Lucas, C., Gould, M., Stanley, B., Brown, G., ... \& Mann, J. (2008).

615 Columbia-Suicide Severity Rating Scale (C-SSRS). New York, NY: Columbia University

$616 \quad$ Medical Center, 2008.

617 Reynolds, C.F., III, Soloff, P.H., Kupfer, D.J., Taska, L.S., Restifo, K., Coble, P.A., \&

McNamara, M.E. (1985). Depression in borderline patients: A prospective EEG sleep study. Psychiatry Research, 14, 1-15.

Ribeiro, J.D., Pease, J.L., Gutierrez, P.M., Silva, C., Bernert, R.A., Rudd, M.D., \& Joiner Jr, T.E. (2012). Sleep problems outperform depression and hopelessness as cross-sectional and longitudinal predictors of suicidal ideation and behavior in young adults in the military. Journal of Affective Disorders, 136(3), 743-750.

Russell, K., Rasmussen, S., \& Hunter, S. C. (2018). Insomnia and nightmares as markers of risk for suicidal ideation in young people: investigating the role of defeat and entrapment. Journal of Clinical Sleep Medicine, 14(5), 775-784.

Sandman, N., Valli, K., Kronholm, E., Vartiainen, E., Laatikainen, T., \& Paunio, T. (2017). Reports, 7(1), 1-7.

Sansone, R.A., Edwards, H.C., \& Forbis, J.S. (2010). Sleep quality in borderline personality disorder: a cross-sectional study. Primary Care Companion to the Journal of Clinical Psychiatry, 12(5): PCC.09m00919.

Selby, E.A. (2013). Chronic sleep disturbances and borderline personality disorder symptoms. Journal of Consulting and Clinical Psychology, 81(5), 941-947.

Selby, E. A., \& Joiner Jr, T. E. (2009). Cascades of emotion: The emergence of borderline 
personality disorder from emotional and behavioral dysregulation. Review of General Psychology, 13(3), 219-229.

Simor, P., \& Horváth, K. (2013). Altered sleep in borderline personality disorder in relation to the core dimensions of psychopathology. Scandinavian Journal of Psychology, 54(4), 300-312.

Sjöström, N., Hetta, J., \& Waern, M. (2009). Persistent nightmares are associated with repeat suicide attempt: a prospective study. Psychiatry Research, 170(2-3), 208-211.

Soloff, P. H., \& Chiappetta, L. (2017). Suicidal behavior and psychosocial outcome in borderline personality disorder at 8-year follow-up. Journal of Personality Disorders, 31 (6), 774-789.

Tkachenko, O., Olson, E. A., Weber, M., Preer, L. A., Gogel, H., \& Killgore, W. D. (2014). Sleep difficulties are associated with increased symptoms of psychopathology. Experimental Brain Research, 232(5), 1567-1574.

Vallières, A., Ivers, H., Bastien, C.H., Beaulieu-Bonneau, S., \& Morin, C.M. (2005). Variability and predictability in sleep patterns of chronic insomniacs. Journal of Sleep Research, 14(4), 447-453.

Wall, K., Vanwoerden, S., Penner, F., Patriquin, M., Alfano, C.A., \& Sharp, C. (2020). Adolescent Sleep Disturbance, Emotion Regulation and Borderline Features in an Inpatient Setting. Journal of Clinical Child \& Adolescent Psychology, 1-15.

Winsper, C., \& Tang, N.K. (2014). Linkages between insomnia and suicidality: prospective associations, high-risk subgroups and possible psychological mechanisms. International Review of Psychiatry, 26(2), 189-204.

Winsper, C., Tang, N.K., Marwaha, S., Lereya, S.T., Gibbs, M., Thompson, A., \& Singh, S.P. 
(2017). The sleep phenotype of Borderline Personality Disorder: A systematic review and meta-analysis. Neuroscience \& Biobehavioral Reviews, 73, 48-67.

661 Wojnar, M., Ilgen, M.A., Wojnar, J., McCammon, R.J., Valenstein, M., \& Brower, K.J. (2009).

662 Sleep problems and suicidality in the National Comorbidity Survey Replication. Journal of Psychiatric Research, 43(5), 526-531.

664 Zanarini, M.C., Frankenburg, F.R., Reich, D.B., \& Fitzmaurice, G.M. (2016). Fluidity of the subsyndromal phenomenology of borderline personality disorder over 16 years of prospective follow-up. American Journal of Psychiatry, 173(7), 688-694.

667 Zuromski, K.L., Cero, I., \& Witte, T.K. (2017). Insomnia symptoms drive changes in suicide ideation: A latent difference score model of community adults over a brief interval. Journal of Abnormal Psychology, 126(6), 739. 


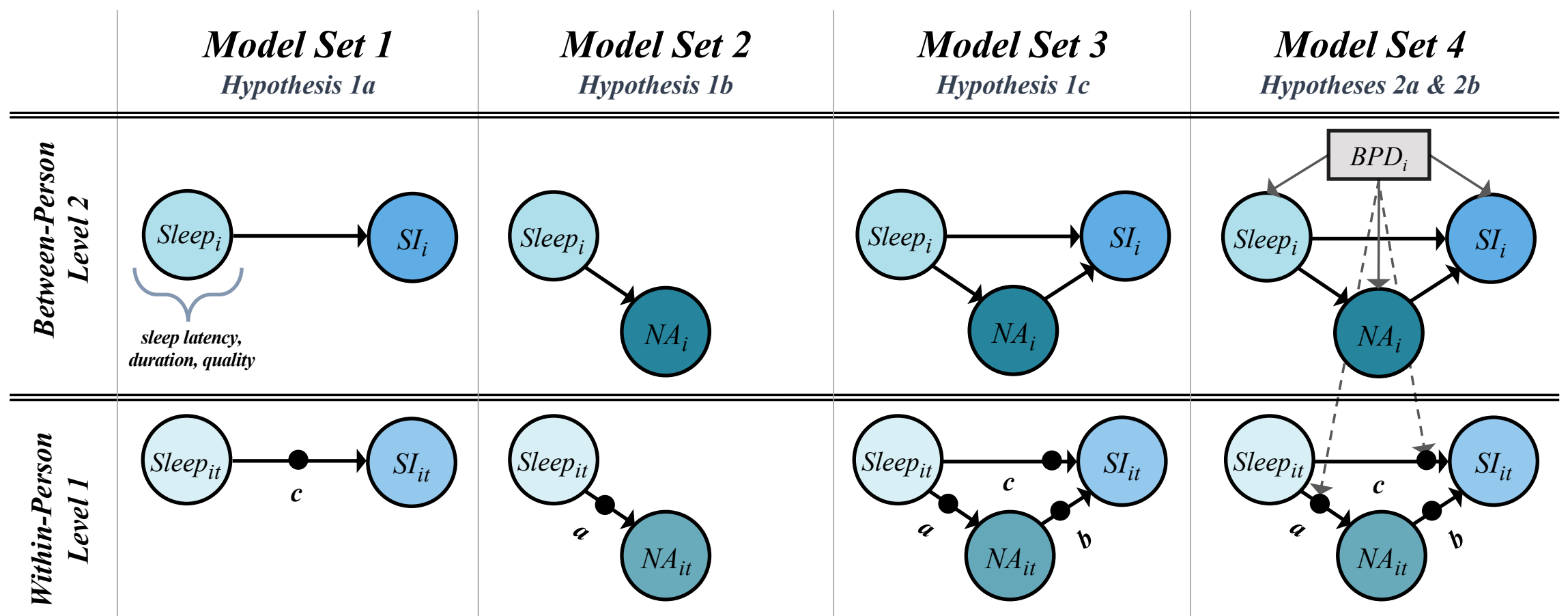

Figure 1. Overview of quantitative model specifications used to test preregistered hypotheses, including decomposition of observed daily variables into between- (subscript $i$ ) and within-person (subscript $t$ ) variance. Note, not all parameters are diagrammed. All models controlled for age and gender at the between-person level, and for time and weekday at the within-person level. Coefficients for covariates are not presented for parsimony. Single headed arrows indicate regression paths. Solid lines represent paths for hypotheses $1 \mathrm{a}-\mathrm{c}$, and dashed for hypotheses $2 \mathrm{a}$ and $\mathrm{b}$.

675 Filled dots represent random effects. NA = negative affect; $\mathrm{SI}=$ suicidal ideation. 
676 Table 1. Correlations among study variables at within and between-person levels.

\begin{tabular}{|c|c|c|c|c|c|c|}
\hline & quality & latency & duration & $\begin{array}{l}\text { negative } \\
\text { affect }\end{array}$ & $\begin{array}{l}\text { suicidal } \\
\text { ideation }\end{array}$ & BPD \\
\hline \multicolumn{7}{|l|}{ within-person } \\
\hline quality & - & & & & & \\
\hline latency & -.34 & - & & & & \\
\hline duration & .30 & -.31 & - & & & \\
\hline negative affect & -.06 & .02 & -.06 & - & & \\
\hline suicidal ideation & -.03 & .07 & -.04 & .15 & - & - \\
\hline \multicolumn{7}{|l|}{$\begin{array}{l}\text { between-person } \\
\text { quality }\end{array}$} \\
\hline latency & -.46 & & & & & \\
\hline duration & .21 & -.14 & & & & \\
\hline negative affect & -.04 & .32 & .10 & & & \\
\hline suicidal ideation & .07 & .30 & .06 & .23 & & \\
\hline BPD & -.52 & .46 & -.12 & .37 & .21 & \\
\hline$M$ & 3.18 & 22.66 & 7.35 & 1.62 & .11 & 39.19 \\
\hline$S D$ & 1.01 & 22.67 & 1.85 & .07 & .04 & 19.50 \\
\hline
\end{tabular}

$677 \quad$ Note. $N=201$ (between), $N=4076$ (within); Bolded values indicate the credibility interval does not contain 678 zero. 
Table 2.

680 Key estimates from three individual multi-level structural equation models showing associations among sleep, negative affect and

681 suicidal ideation.

\begin{tabular}{|c|c|c|c|c|c|c|}
\hline & \multicolumn{2}{|c|}{$\begin{array}{c}\text { Model Set } 1 \\
\text { Hypothesis 1a }\end{array}$} & \multicolumn{2}{|c|}{$\begin{array}{c}\text { Model Set } 2 \\
\text { Hypothesis } 1 \mathrm{~b}\end{array}$} & \multicolumn{2}{|c|}{$\begin{array}{c}\text { Model Set } 3 \\
\text { Hypothesis 1c }\end{array}$} \\
\hline & Est $(\mathrm{CI})$ & $\beta$ & $\operatorname{Est}(\mathrm{CI})$ & $\beta$ & Est $(\mathrm{CI})$ & $\beta$ \\
\hline \multicolumn{7}{|c|}{ sleep latency } \\
\hline \multicolumn{7}{|l|}{ within-person } \\
\hline sleep $\rightarrow$ suicidal ideation (c) & $.00[-.00, .01]$ & .14 & - & - & $.00[00, .00]$ & .13 \\
\hline sleep $\rightarrow$ NA (a) & - & - & $.00[-.00, .01]$ & .06 & $.00[-.00, .00]$ & .02 \\
\hline NA $\rightarrow$ suicidal ideation (b) & - & - & - & - & $.14[.08, .20]$ & .45 \\
\hline indirect effect & - & - & - & - & $.00[-.00,-.01]$ & - \\
\hline \multicolumn{7}{|l|}{ between-person } \\
\hline sleep $\rightarrow$ suicidal ideation & $.01[.00, .01]$ & .24 & - & - & $.99[.38,1.70]$ & .24 \\
\hline sleep $\rightarrow$ NA & - & - & $.02[.01, .02]$ & .44 & $3.92[2.50,5.60]$ & .44 \\
\hline $\mathrm{NA} \rightarrow$ suicidal ideation & - & - & - & - & $.06[.04, .10]$ & .36 \\
\hline \multicolumn{7}{|c|}{ sleep duration } \\
\hline \multicolumn{7}{|l|}{ within-person } \\
\hline sleep $\rightarrow$ suicidal ideation (c) & $-.00[-.01, .01]$ & -.04 & - & - & $-.01[-02, .01]$ & -.07 \\
\hline sleep $\rightarrow$ NA (a) & - & - & $-.01[-.02, .01]$ & -.10 & $-.02[-03, .00]$ & -.15 \\
\hline NA $\rightarrow$ suicidal ideation $(b)$ & - & - & - & - & $.15[.09, .20]$ & .48 \\
\hline indirect effect & - & - & - & - & $.00[-.01, .01]$ & - \\
\hline \multicolumn{7}{|l|}{ between-person } \\
\hline sleep $\rightarrow$ suicidal ideation & $-.02[-.03, .07]$ & .06 & - & - & $.01[-.04, .07]$ & .04 \\
\hline sleep $\rightarrow$ NA & - & - & $-.03[-.12, .06]$ & -.07 & $-.04[-.16, .08]$ & -.05 \\
\hline NA $\rightarrow$ suicidal ideation & - & - & - & - & $.07[.04, .10]$ & .37 \\
\hline
\end{tabular}




\begin{tabular}{|c|c|c|c|c|c|c|}
\hline \multicolumn{7}{|c|}{ subjective sleep quality } \\
\hline sleep $\rightarrow$ suicidal ideation (c) & $-.01[-.03, .00]$ & -.16 & - & - & $-.01[-.03, .00]$ & -.21 \\
\hline sleep $\rightarrow$ NA (a) & - & - & $-.05[-.10, .-02]$ &.- .33 & $-.08[-.11,-.05]$ & -.49 \\
\hline NA $\rightarrow$ suicidal ideation (b) & - & - & - & - & $.13[.08, .19]$ & .45 \\
\hline indirect effect & - & - & - & - & $-.01[-.02, .01]$ & - \\
\hline \multicolumn{7}{|l|}{ between-person } \\
\hline sleep $\rightarrow$ suicidal ideation & $-.03[-.10, .03]$ & -.06 & - & - & $.00[-.03, .03]$ & .00 \\
\hline sleep $\rightarrow \mathrm{NA}$ & - & - & $-.24[-.37, .-12]$ & -.29 & $-.13[-.20,-.07]$ & -.32 \\
\hline NA $\rightarrow$ suicidal ideation & - & - & - & - & $.07[.04, .10]$ & .36 \\
\hline
\end{tabular}

683 Note. $N=201$ (between), $N=4076$ (within); $\rightarrow$ indicates regression; $\beta$ parameter estimates are standardized. $95 \%$ credibility intervals of

684 unstandardized parameter estimates are in parentheses. Bolded values indicate the credibility interval does not contain zero; NA = negative affect. 
685 Table 3.

686 Key estimates from multilevel models with sleep predicting suicidal ideation or negative affect

687 and moderation of these within-person effects by BPD severity.

\begin{tabular}{|c|c|c|c|c|}
\hline & \multicolumn{4}{|c|}{ Model Set 4} \\
\hline & \multicolumn{2}{|c|}{ Hypothesis $2 \mathrm{a}$} & \multicolumn{2}{|c|}{ Hypothesis $2 b$} \\
\hline & Est (CI) & $\beta$ & $\operatorname{Est}(\mathrm{CI})$ & $\beta$ \\
\hline \multicolumn{5}{|l|}{ sleep latency } \\
\hline BPD $\rightarrow$ sleep & $.31[.21, .42]$ & .42 & $.32[.22, .43]$ & .42 \\
\hline $\mathrm{BPD} \rightarrow \mathrm{NA}$ & - & - & $.02[.01, .02]$ & .48 \\
\hline $\mathrm{BPD} \rightarrow$ suicidal ideation & $.00[.00,00]$ & .14 & - & - \\
\hline BPD x (sleep $\rightarrow$ suicidal ideation) & $.00[.00, .00]$ & .06 & - & - \\
\hline BPD x (sleep $\rightarrow$ NA) & - & - & $.00[.00, .00]$ & -.01 \\
\hline \multicolumn{5}{|l|}{ sleep duration } \\
\hline BPD $\rightarrow$ sleep & $-.01[-.02, .01]$ & -.09 & $-.01[-.02 .00]$ & -.12 \\
\hline $\mathrm{BPD} \rightarrow \mathrm{NA}$ & - & - & $.02[.01 .02]$ & .58 \\
\hline $\mathrm{BPD} \rightarrow$ suicidal ideation & $.00[.00, .01]$ & .22 & - & - \\
\hline BPD x (sleep $\rightarrow$ suicidal ideation) & $.00[-.00, .00]$ & -.05 & - & - \\
\hline BPD x (sleep $\rightarrow$ NA) & - & - & $-.00[.00 .00]$ & -.10 \\
\hline $\mathrm{BPD} \times(\mathrm{NA} \rightarrow$ suicidal ideation $)$ & - & - & - & - \\
\hline \multicolumn{5}{|l|}{ subjective sleep quality } \\
\hline $\mathrm{BPD} \rightarrow$ sleep & $-.02[-.02,-.01]$ & -.50 & $-.02[-.02,-.01]$ & -.50 \\
\hline $\mathrm{BPD} \rightarrow \mathrm{NA}$ & - & - & $.02[.01, .02]$ & .56 \\
\hline $\mathrm{BPD} \rightarrow$ suicidal ideation & $.01[.00, .01]$ & .25 & - & - \\
\hline BPD x (sleep $\rightarrow$ suicidal ideation) & $.00[-.00, .00]$ & -.10 & - & - \\
\hline BPD x (sleep $\rightarrow$ NA) & - & - & $-.00[-.00, .00]$ & -.15 \\
\hline BPD $x$ (NA $\rightarrow$ suicidal ideation) & - & - & - & - \\
\hline
\end{tabular}

688 Note. $N=201$ (between), $N=4076$ (within); $\rightarrow$ indicates regression; $\beta$ parameter estimates are

689 standardized. 95\% credibility intervals of unstandardized parameter estimates are in parentheses. Bolded

690 values indicate the credibility interval does not contain zero; NA = negative affect. 